Apidologie, 1979, 10 (1), 3-15.

\title{
PRODUCTION DE MIEL \\ CHEZ DES HYBRIDES INTERRACIAUX D'ABEILLES \\ (APIS MELLIFICA L.) \\ LORS DE GÉNÉRATIONS SUCCESSIVES \\ DE RÉTROCROISEMENT SUR LA RACE LOCALE.
}

\author{
Honigproduktion bei Rassenhybriden der Honigbiene (Apis mellifica L.) \\ in aufeinanderfolgenden Generationen der Rückkreuzung mit der lokalen Rasse
}

\author{
J. M. CORNUET, J. FRESNAYE \\ avec la collaboration technique de J. BLANC et R. PARIS \\ Station Expérimentale d'Apiculture, I.N.R.A. \\ F-84I40 MONTFAVET
}

SUMMARY

HONEY YIELD IN INTERRACIAL HONEYBEE

(APIS MELLIFICA L.) HY'BRIDS

DURING SUCCESSIVE GENERATIONS

OF BACKCROSSES TO THE LOCAL RACE

\begin{abstract}
In order to look at the honey yield of (ligustica $\times$ caucasica) $\times$ mellifica hybrids when they are backcrossed to mellifica honeybees during two generations, four strains have been created. The results show a most important variability between these lines that leads to hide the differences between generations. Yet, the $F_{1}$ of the best strain is significantly higher than the reference population. On the other hand, the mean $\mathrm{F}_{3}$ is distinctly below.

This work reveals the necessity of practising a real selection for combining ability and the advantage of replacing hybrid queens before the $F_{3}$.
\end{abstract}

\section{RÉSUMÉ}

Afin de suivre le rendement en miel chez les hybrides (ligustica $\times$ caucasica) $\times$ mellifica lors de générations successives de croisement en retour sur le parent mellifica, quatre lignées ont été créées. Les résultats montrent une très grande variabilité entre lignées qui tend à masquer les différences entre générations. Cependant, la $F_{1}$ de la meilleure lignée est significativement supérieure au tèmoin. Par contre. d'une manière générale la $F_{3}$ lui est nettement inférieure.

Ce travail révèle la nécessité d'une sélection effective pour la valeur en croisement et l'avantage de remplacer les reines hybrides avant la $\mathrm{F}_{3}$. 


\section{INTRODUCTION}

Depuis plus d'un demi-siècle, de nombreuses tentatives de sélection de l'Abeille ont été engagées dans divers pays. Les travaux les plus anciens présentent généralement peu d'intérêt reposant sur des postulats génétiques simplistes et parfois erronés. D'une manière générale, on peut constater avec RUTTNER (1968) que les résultats visibles de cette sélection sont assez faibles du moins en ce qui concerne la récolte de miel. Il y a à cela plusieurs explications. Il faut tout d'abord considérer l'empirisme des méthodes de sélection utilisées chez l'Abeille en comparaison de celles appliquées chez les autres espèces domestiques. Les études théoriques y ont été extrêmement limitées en grande partie à cause des difficultés particulières à la biologie de cette espèce. D'autre part, l'augmentation de la consanguinité entraînée par la sélection est plus délétère chez l'Abeille que chez tout autre animal domestique en raison de l'existence d'allèles d'incompatibilité (MACKensen et Roberts 1951, Woyke 1963-1972, MAul 1972). Nous avons montré (FRESNAYE 1966) que des lignées maintenues en étroite consanguinité ne pouvaient survivre sans secours artificiel au-delà de deux générations. AvETISIAN et TCHEREVKo (1971) ont en outre démontré que la consanguinité réduit le nombre des ovarioles chez les reines.

Devant ces difficultés, les sélectionneurs se sont rapidement tournés vers la création d'hybrides entre races. RUTTNER (1967) a résumé les résultats obtenus en Europe avec des croisements des races ligustica, mellifica, caucasica, carnica et cypria. Ces résultats sont très variables, l'augmentation de récolte est parfois faible, parfois elle atteint 100 à $200 \%$. Nous avons pour notre part (FRESNAYE 1966) enregistré des récoltes représentant $253 \%$ de celles des témoins de race pure avec des hybrides (ligustica $\times$ mellifica) $\times$ mellifica.

Il arrive également que l'hétérosis ne se manifeste pas du tout, comme dans les croisements cypria $\times$ carnica, cypria $\times$ mellifica ou ligustica $\times$ carnica. Par ailleurs RUTTNER (1968) fait remarquer que les différentes souches d'une même race montrent des aptitudes au croisement très variables. Nous avons nous-mêmes constaté ce phénomène chez des hybrides ligustica $\times$ mellifica (FRESNAYE 1966). Après des essais prélimiriaires très encourageants, nous avons effectué de 1970 à 1975 (FrESNAYE, LAviE, BoESIGER 1974 - FresNAYE, LAVIE 1976) des expériences systématiques faisant intervenir des hybrides triples (ligustica $\times$ caucasica) $\times$ mellifica. Comparativement à des témoins de race pure mellifica, la récolte varie de $157 \%$ à $361 \%$ avec une moyenne de $229 \%$ calculée sur 209 ruches et sur une période de six années. D’autres auteurs signalent des augmentations importantes de récoltes grâce à divers types d'hybrides (BILASH, Makarov et Sedikk 1976 - Melnitchenko et Trichina 1976 - Velitchkov 1976 RAGHIM-ZADE 1976).

Tous ces résultats montrent l'intérêt des hybridations entre races pour l'amélioration de la productivité des colonies d'abeilles. Sur le plan pratique, l'utilisation d'hybrides nécessite toutefois des soins particuliers. En effet, il est généralement admis que 
lors des générations successives de rétrocroisement des hybrides sur la race locale la production chute rapidement. En fait, cette assertion repose au moins autant sur des notions théoriques que sur des résultats expérimentaux. C'est pourquoi il nous est apparu nécessaire de contrôler la justesse de cette hypothèse dans le cas particulier de notre hybride trois voies en suivant son évolution lors des rétrocroisements sur la race mellifica.

De plus, pour évaluer la variabilité de l'aptitude au croisement de diverses souches et rendre ainsi nos conclusions plus générales, il a été decidé de constituer quatre lignées différentes.

\section{MATÉRIELS ET MÉTHODES}

\section{1. - Cheptel et méthodes apicoles}

Les essais ont été effectués à la Station Expérimentale d'Apiculture de l'I.N.R.A. à Montfavet. Les mesures des caractères morphologiques des abeilles effectuées selon la méthode décrite par FresNAYE (1974) ont permis de s'assurer de l'appartenance des géniteurs aux races Apis mellifica ligustica, Apis mellifica caucasica et Apis mellifica mellifica.

Toutes les reines proviennent d'élevages artificiels selon la méthode du "double transfert de larves " utilisée à la Station depuis 1965 et décrite par Fresnaye (1975).

Les ruchers expérimentaux hivernent dans les Alpilles afin d'obtenir un développement précoce des colonies au moment de la floraison du romarin. Ils sont ensuite transhumés dans la Crau pour la récolte de miel de printemps sur les prairies naturelles. Au mois de juillet, les ruchers sont transportés entre le plateau de Vaucluse et le Massif du Ventoux pour la récolte des miels de lavande et lavandin qui est généralement la plus importante dans notre région. Dans nos conditions d'expérimentation, nous n'effectuons pas autant de transhumances que les apiculteurs professionnels. Il faudra en tenir compte lors de comparaisons globales de nos récoltes de miel avec des récoltes obtenues en d'autres circonstances et en d'autres lieux.

Les divers groupes en expérimentation sont répartis en nombre sensiblement égal en deux ruchers. Les colonies sont disposées au hasard dans ces ruchers et des précautions sont prises pour éviter la " dérive " des butineuses susceptible d'entrainer de grossières erreurs dans le calcul du rendement en miel (FrESNAYE 1963). Toutes les colonies suivent les mêmes déplacements.

Le "rendement " en miel d'une colonie est parfois très différent de la "récolte " de miel car divers faćteurs interviennent pouvant fausser les comparaisons. Le rendement est calculé selon la formule suivante (FRESNAYE et al. 1974):

$$
R=\left(R_{1}+R_{2} \ldots+H_{2}\right)-\left(H_{1}+N\right)
$$

où

$R_{1}, R_{2}, \ldots$ représentent les récoltes de l'année.

$\mathrm{H}_{1}$ et $\mathrm{H}_{2}$ sont les poids de la ruche avant l'hivernage respectivement l'année précédant l'expérience et l'année de l'expérience.

$\mathrm{N} \quad$ est le poids du sucre de nourrissement.

Aucune mesure de contrôle n'est effectuée au cours de l'année de l'élevage des reines et de la création des colonies, c'est-à-dire avant la mesure $\mathrm{H}_{1}$.

\section{2 - Méthodes statistiques}

Plusieurs types d'analyses de variance ont été effectuées : comparaison de deux moyennes, analyse d'un tableau à deux voies de classification, comparaison multiple de moyennes. L'ensemble de ces analyses 

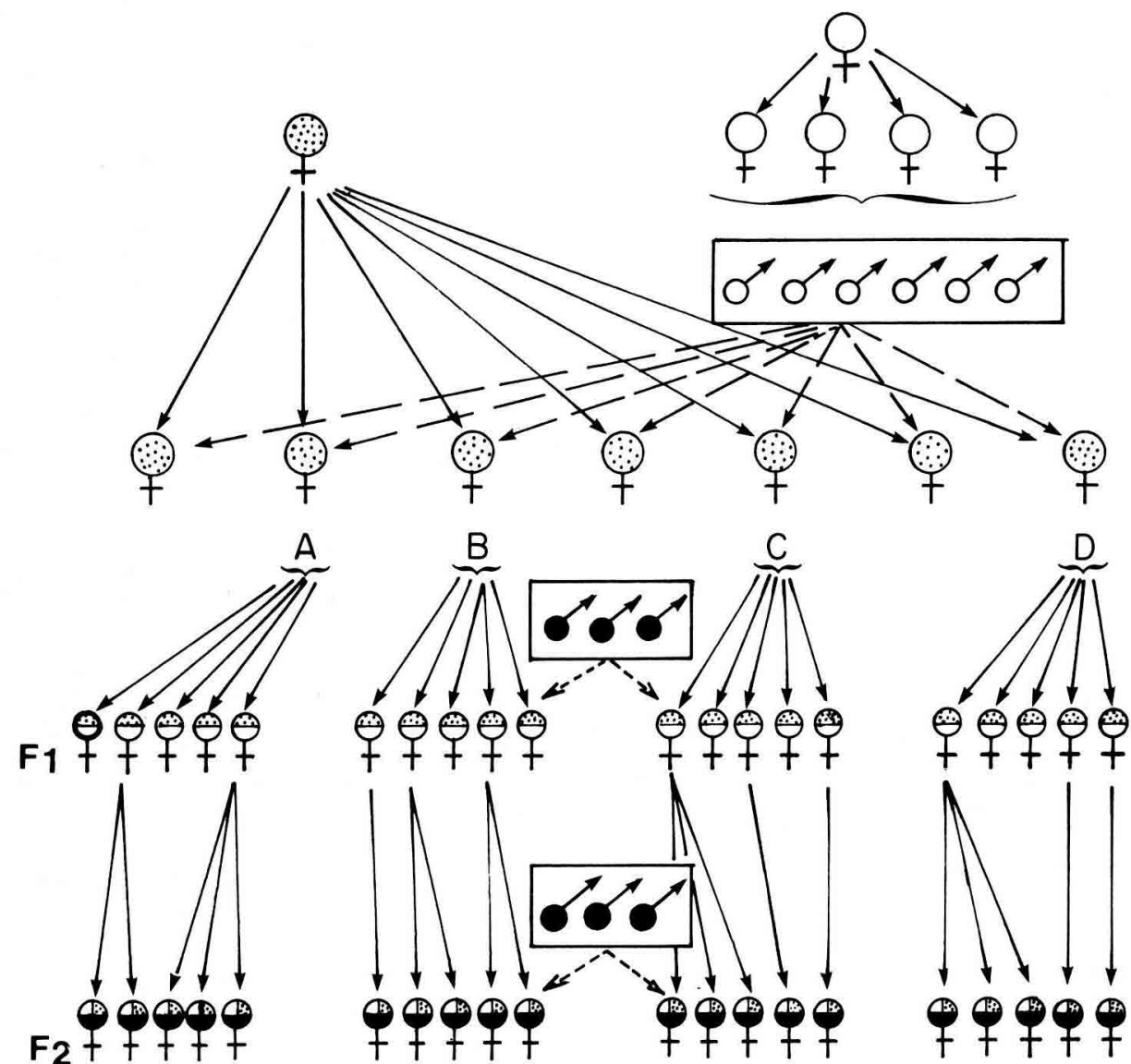

$\mathrm{F}_{2} \mathrm{~T} F \mathrm{Ft}$
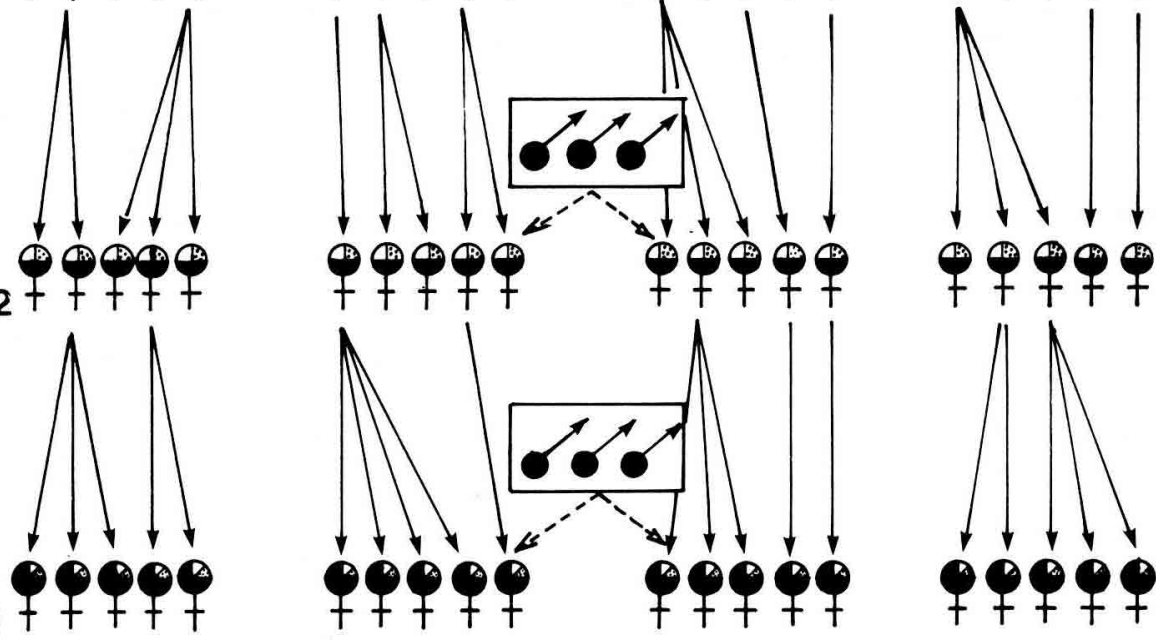

F3 $+7+7$

$q$ reine caucasica $\$$ reine mère

$\$$ reine ligustica $q$ reine fille

○ caucasica Königin $\stackrel{P}{\ddagger}$ Mutter Königin

$\$$ ligustica Königin $\dot{q}$ Tochter Königin

$\sigma^{\prime}$ mâle caucasica _. $\sigma^{\infty}$ insémination artificielle $\sigma^{-}$caucasica Drohne _.. $\sigma^{\prime \prime}$ instrumentelle Besamung

$\checkmark$ mâle melifica $\quad . \sigma^{\prime}$ fécondation naturelle $\sigma^{\prime}$ mellifica Drohne $\sigma^{\varnothing}$ natürliche Begattung

FIG. 1. - Généalogie des reines des colonies soumises à l'expérience.

Авв. 1. - Abstammung der Königinnen in den Versuchsiölkern. 
suppose réunies au moins les deux conditions suivantes : normalité des données, égalité des variances résiduelles. $A$ priori, il n'y a aucune raison de soup̧̧onner la première condition de n'être pas remplie en raison du caractère même de la variable " rendement ". Par contre le petit nombre de données associé à leuı grande variabilité ne permet pas de se faire une idée très précise concernant la seconde condition. Pour ces deux dernières raisons, très peu de différences s'avèreront significatives même au seuil de $10 \%$ qu'il nous est apparu judicieux de considérer ici toujours pour ces mêmes raisons. L’analyse du tableau à deux voies de classification et la comparaison multiple qui lui fait suite ont été réalisées sur l'ordinateur de l'I.N.R.A. de Toulouse à l'aide des programmes principaux ANVARM et BONFER. En effet, l'inégalité des effectifs à l'intérieur des cases du tableau rend les calculs assez complexes. Précisons que BONFER réalise une comparaison multiple selon la méthode de BONFERONNI (répartition du risque de $1^{\text {re }}$ espèce entre toutes les comparaisons possibles).

\section{3. - Généalogie des colonies expérimentées}

Le schéma généalogique des lignées d'hybrides interraciaux (ligustica $\times$ caucasica) $\times$ mellifica est représenté à la figure 1. Les lignées des races ligustica et caucasica conservées à la Station sont maintenues " pures" (FrESNAYE et al. 1974) par insémination artificielle et en n introduisant qu'un taux de consanguinité aussi faible que possible par des croisements de diverses lignées de même race.

Dans ce travail, nous avons effectué un élevage de reines issues d'une colonie ligustica. Ces reines, sœurs ou demi-sœurs, sont fécondées par insémination artificielle par des mâles issus de quelques colonies caucasica dont les reines sont filles d'une même mère. Leurs reines filles sont donc des hybrides $F_{1}$ (ligustica $\times$ caucasica). Par tirage au sort on en constitue quatre groupes, chaque groupe étant une fratrie issue d'une reine ligustica fécondée par des mâles caucasica (reine souche).

Les reines $F_{1}$ des quatre groupes ou lignées sont élevées simultanément, la répartition sur les ruches éleveuses se faisant selon le schéma suivant : chacune des quatre ruches éleveuses reçoit les larves des quatre souches $\mathrm{A}, \mathrm{B}, \mathrm{C}$ et $\mathrm{D}$ réparties également sur deux barettes porte-cupules et repérées par des punaises colorées; ceci vise à supprimer un éventuel effet de la ruche éleveuse. Ces reines sont fécondées naturellement en rucher de fécondation isolé. Lorsqu'elles ont commencé à pondre, elles deviennent à leur tour les géniteurs des reines $F_{2}$ qui débutent la série des "backcrosses" sur la race locale. Celles-ci sont élevées et fécondées dans les mêmes conditions que les $F_{1}$ et constituent les mères des reines $F_{3}$ auxquelles est appliqué la même méthode. Le processus de création de ces trois générations est simultané pour les quatre lignées et s'étale tout au long de la période d’élevage qui va de la fin de l'hiver au début de l'été, la même année. Fin juillet, toutes les colonies dont les reines sont $F_{1}, F_{2}$ ou $F_{3}$ sont constituées en vue d'être testées l'année suivante. Parallèlement, un groupe de colonies témoins est joint aux hybrides. Il est constitué de colonies dont les reines de race mellifica ont le même âge que les reines hybrides et sont issues de plusieurs colonies prises au hasard parmi les géniteurs de cette race.

\section{RÉSULTATS}

Le tableau 1 fournit l'ensemble des résultats par lignée et par génération. Dans chaque case, les rendements de chaque colonie exprimés en $\mathrm{kg}$ constituent la colonie de gauche tandis qu'à droite on note de haut en bas le nombre de colonies, le total de leurs rendements et la moyenne de leurs rendements. Ces trois dernières statistiques se retrouvent dans la dernière colonne (résultats globaux par génération) et la dernière ligne (résultats globaux par lignée), la dernière case rassemblant les statistiquesde toutes les colonies de l'expérimentation témoins non compris. Les rendements des colonies témoins sont reportés au tableau II. 
TABL. 1. - Rendements en $\mathrm{kg}$ des colonies classées par lignée et pas génération (cf. texte).

ТАв. 1. - Ertrag der Völker in kg, klassifiziert nach Linie und nach Generation (s. Text).

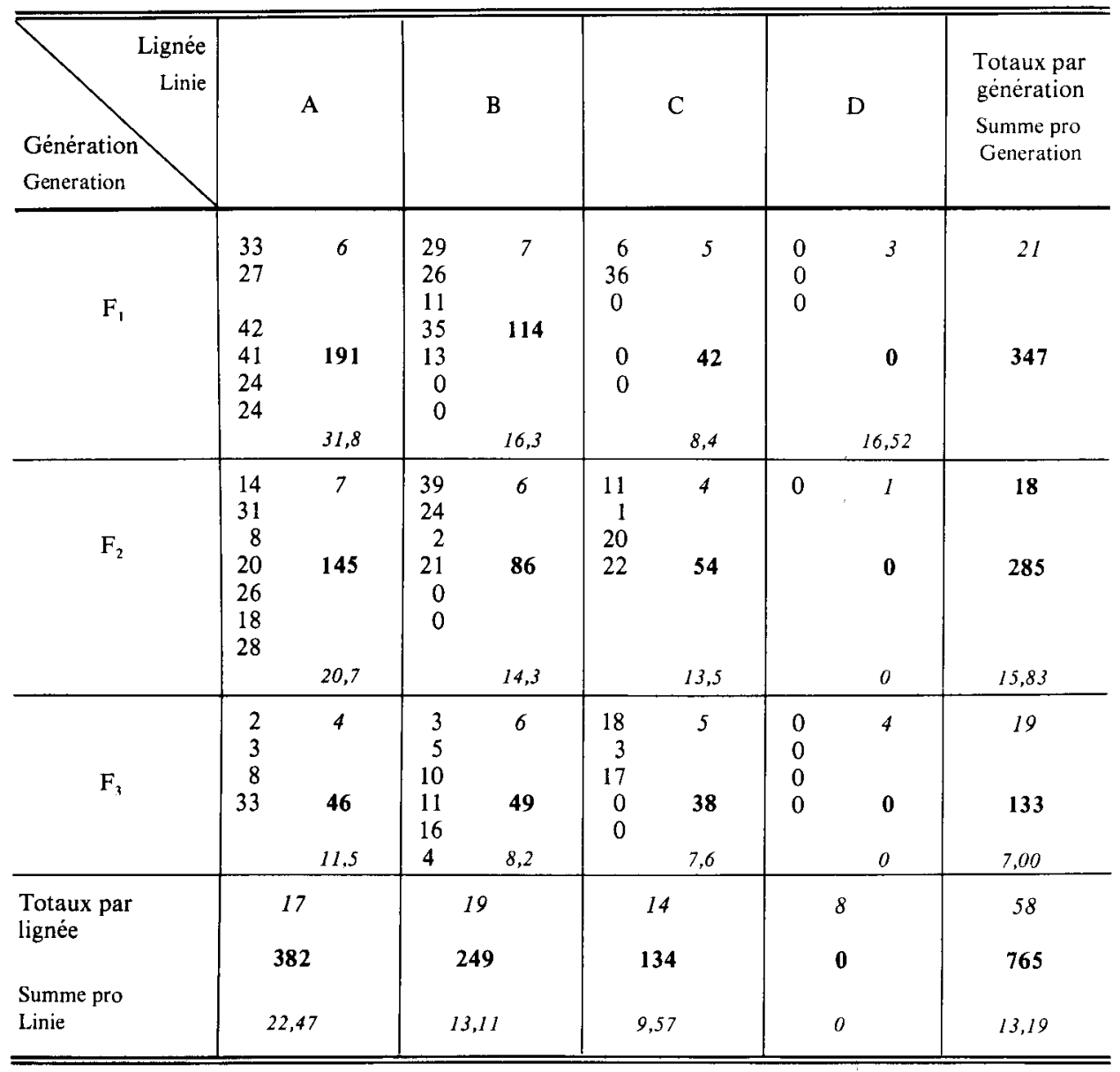

TABL. 2. - Rendements en $\mathrm{kg}$ des colonies témoins.

TAB. 2. - Ertrag der Kontrollvölker in $\mathrm{kg}$.

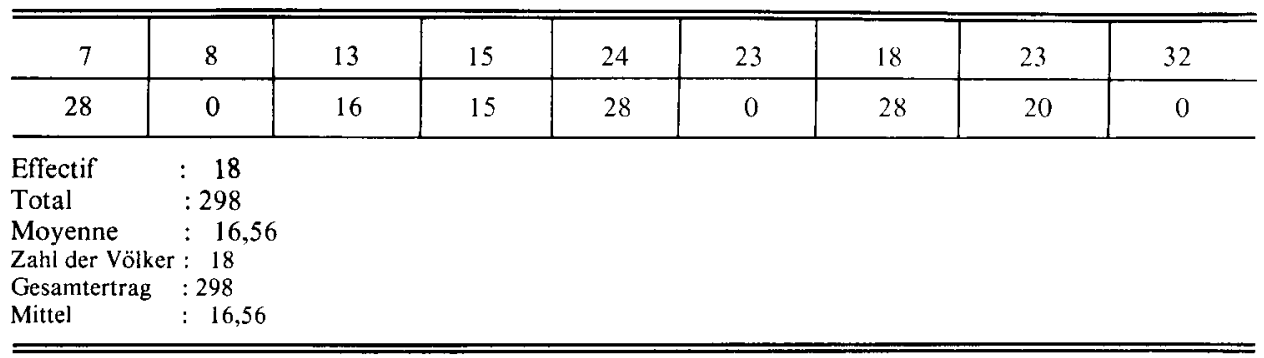




\section{1. - Comparaison de chaque génération avec le témoin}

C'est la question principale et qui a motivé cette recherche.
a) $F_{1}$ - témoin

Les moyennes pour la $F_{1}(16,52)$ et pour le témoin $(16,56)$ sont suffisamment proches pour que toute analyse statistique soit superflue : ces moyennes ne diffèrent pas significativement.

b) $F_{2}-t e ́ m o i n$

Là aussi, les deux moyennes $F_{2}(15,83)$ et témoin $(16,56)$ ne diffèrent pas de façon significative.

c) $F_{3}-$ témoin

La moyenne de la $\mathrm{F}_{3}(7,00)$ diffère significativement $(P<0,01)$ de celle des témoins (Cf. tableau III).

TABL. 3. - Comparaison $F_{3}$ témoin.

TAB. 3. - Vergleich $F_{3}$ - Kontrolle.

\begin{tabular}{l|c|c|c|c}
\hline \multicolumn{1}{c|}{$\begin{array}{c}\text { Origine de la variation } \\
\text { Ursache der Variation }\end{array}$} & ddl & SCE & SCM & F \\
\hline $\begin{array}{l}F_{i} \text { - témoin } \\
F_{1} \text { - Kontrolle } \\
\begin{array}{l}\text { Résiduelle } \\
\text { Rest }\end{array}\end{array}$ & 1 & 843,99 & 843,99 & $9,31^{* *}$ \\
\hline
\end{tabular}

** Cf, légende tableau $\mathrm{V}$.

s. Eirklärung zu Tab. 5.

Il apparait donc que seule la $F_{3}$ se distingue du témoin et cela, d'une façon très nette.

$\mathrm{Ce}$ résultat est surprenant car les études antérieures citées plus haut laissaient attendre une $F_{1}$ significativement supérieure au témoin. Mais la consultation du tableau I des données fait apparaitre un comportement disparate des diverses lignées. Il nous faut donc faire l'analyse statistique de ce tableau en prenant en compte les deux facteurs "générations" et "lignées".

\section{2. - Analyse du tableau "générations $\times$ lignées"}

La première étape de l'analyse (tableau IV) montre qu'il n'y a pas d'intéraction significative entre les deux facteurs, qu'il n'y a pas d'effet générations significatif, mais qu'il y a un effet lignées très significatif $(P<0,01)$. Cela n'est pas surprenant puisqu'une lignée ne possède que des colonies à rendement nul. L'existence de cette dernière 
TABL. 4. - Analyse de variance du tableau "Générations $\times$ lignées ".

TAB. 4. - Varianzanalyse der Tabelle "Generationen $\times$ Linien".

\begin{tabular}{l|c|c|c|c}
\hline \hline \multicolumn{1}{c|}{$\begin{array}{c}\text { Origine de la variation } \\
\text { Ursache der Variation }\end{array}$} & $\mathrm{ddl}$ & $\mathrm{SCE}$ & $\mathrm{SCM}$ & $\mathrm{F}$ \\
\hline $\begin{array}{l}\text { Générations } \\
\text { Generationen }\end{array}$ & 2 & 632,72 & 316,36 & $2,61 \mathrm{NS}$ \\
$\begin{array}{l}\text { Lignées } \\
\text { Linien }\end{array}$ & 6 & 2585,01 & 861,67 & $7,11^{* *}$ \\
$\begin{array}{l}\text { Intéraction } \\
\text { Interaktion } \\
\begin{array}{l}\text { Résiduelle } \\
\text { Rest }\end{array}\end{array}$ & 46 & 5574,26 & 121,18 & $0,98 \mathrm{NS}$ \\
\hline
\end{tabular}

Cf. légende tableau V.

s. Erklärungen zu Tab. 5.

" écrase " en quelque sorte les différences entre générations et masque un quelconque effet du facteur "génération ".

La seconde étape qui se déduit des résultats de la première consiste en la comparaison 2 à 2 des lignées. Celle-ci apparait au tableau $\mathrm{V}$ et si l'on classe les lignées selon

TABL. 5. - Comparaison des lignées 2 à 2 .

ТАв. 5. - Vergleich der Linien $2 z u 2$.

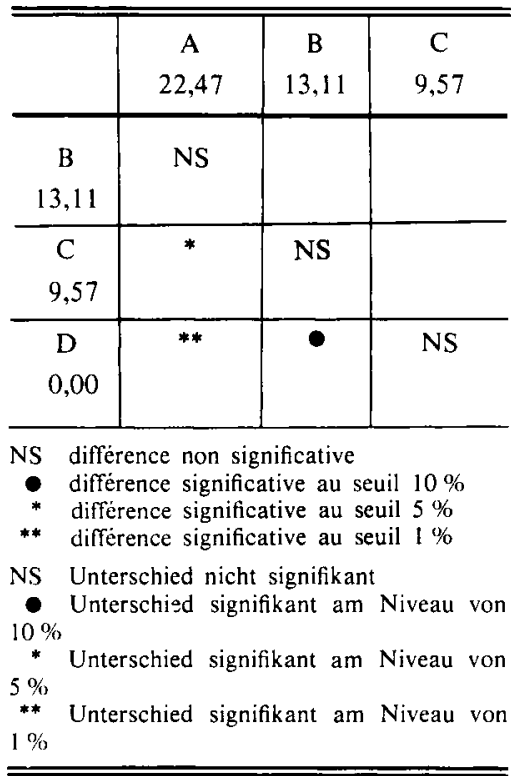


leur production moyenne, seules les différences entre lignées consécutives ne sont pas significatives.

Puisque les lignées ont des comportements différents, il semble intéressant de les comparer séparément au témoin.

\section{3. - Comparaison de chaque lignée}

avec le témoin génération par génération

Les résultats sont indiqués au tableau VI. Les seules différences significatives concernent les lignées A et $\mathrm{D}$. Les performances nulles de la lignée $\mathrm{D}$ expliquent cette différence et tout autre commentaire est superflu à ce niveau. Seule la $F_{1}$ de la lignée $A$ surpasse le témoin. Sur quatre lignées c'est la seule dont la $F_{1}$ fournit le résultat habituel c'est-à-dire $200 \%$ des témoins, environ. Notons que dans cette lignée les $F_{2}$ et $F_{3}$ ne sont pas significativement différentes du témoin. Pour la $F_{3}$, la conclusion doit être prise avec beaucoup de précautions, étant donné le petit nombre de valeurs d'une part et la présence d'une donnée qui semble aberrante.

TABL. 6. - Comparaison de chaque lignée al'ec le témoin, génération par génération

TAв. 6. - Vergleich jeder Linie mit der Kontrolle Generation für Generation

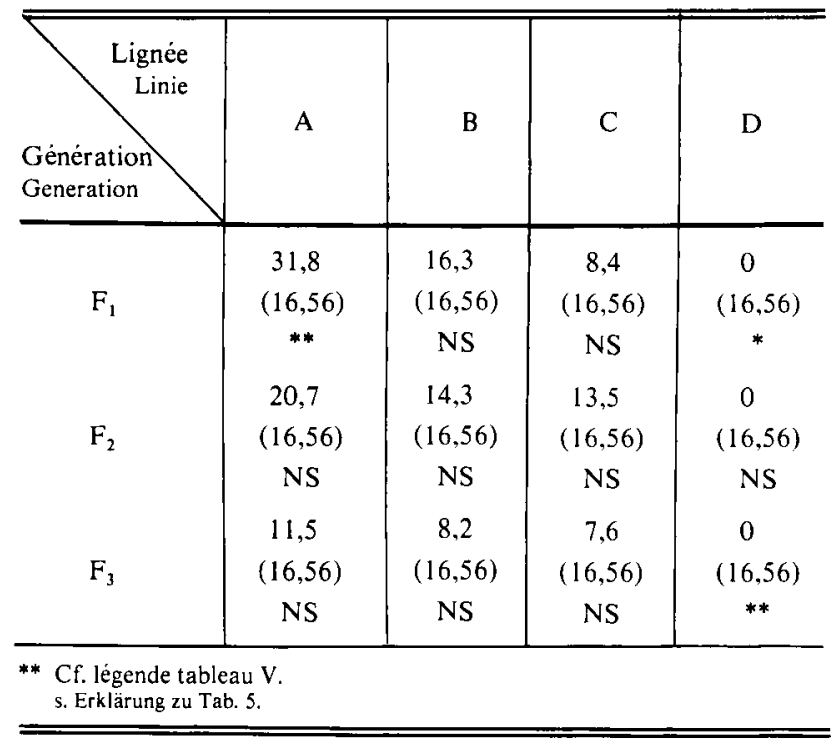

Ces mêmes raisons (faible nombre et variabilité des données) sont à l'origine du manque de signification pour les lignées $B$ et $C$. En regroupant la $F_{3}$ des lignées $A, B$ et $\mathrm{C}$ on trouve une moyenne de 8,87 significativement inférieure $(P<0,05)$ à celle des témoins. 


\section{DISCUSSION}

La question que nous nous posions au départ était la suivante : quel est le comportement d'hybrides interraces lors de rétrocroisements successifs sur la race locale? Par précaution, nous avions pris soin de créer quatre lignées hybrides afin de détecter d'éventuelles différences entre ces lignées. En fait, il s'est avéré dans notre expérimentation que le facteur " lignée " a été bien plus discriminant que le facteur "génération ". Les quatre lignées ont un comportement en $F_{1}$ qui varie de l'hybride à forte hétérosis (A) à l'échec complet (D) avec les niveaux intermédiaires (B et C). Ce résultat est en contradiction avec tous ceux obtenus précédemment avec de tels hybrides qui manifestaient une qualité et une homogénéité supérieure (FrESNAYE et al. 1974; FresNAYE et LAVIE, 1976). Aussi faut-il examiner toutes les possibilités d'artefacts qui seraient à l'origine d'une baisse de qualité chez certaines lignées seulement, en particulier la lignée D. En fait, aucune lignée n'a fait l'objet d'un traitement séparé, ni lors de l'élevage des reines (toutes les reines d'une même génération sont élevées simultanément, avec la même méthode du double greffage et avec une répartition égale entre ruches éleveuses), ni lors de la fécondation (il n'y a qu'un seul rucher de fécondation), ni lors de l'introduction des reines dans les essaims (la répartition a été faite au hasard), ni lors de la conduite des ruches. Il semble donc que nous ayons pris le maximum de précautions pour éviter ce genre d'artefact et le résultat obtenu, pour inhabituel qu'il soit, nous apparaît néanmoins significatif et il importe à présent de tenter de l'expliquer.

La première idée est une différenciation génétique entre les lignées. Cependant les reines $F_{1}$ des différentes lignées sont apparentées puisqu'elles ont même "grand-mère " maternelle (la colonie mère ligustica) et même "grand-mère" paternelle (la colonie mère caucasica). Ceci leur confère une parenté au moins égale à celle de cousins germains chez les espèces diploïdes habituelles $(\varphi=1 / 16)$. Il semble donc difficile d'expliquer une telle variabilité uniquement par des différences d'origine génétique. Toutefois il ne faut pas écarter l'hypothèse de l'apparition en $F_{1}$ de mutations délétères notamment chez les reines de la lignée $\mathrm{D}$, celles-ci pouvant être à hérédité chromosomique ou plus vraisemblablement cytoplasmique puisque la descendance est uniquement matricline et est affectée en totalité. Pendant les trois jours qui s'écoulent de la ponte de l'œuf à son éclosion, le développement de l'embryon issu de la fusion des noyaux gamétiques s'effectue au dépend des réserves vitellines accumulées lors de l'évolution de l'oocyte (Nelson 1915 cité dans SNODGRass 1956). On peut envisager qu'une qualité ou une quantité insuffisante de ces réserves puisse affecter le développement de l'insecte et entraîner des déficiences dans une ou plusieurs de ses fonctions lors de sa vie adulte. L'origine de cette carence peut se situer au niveau chromosomique ou cytoplasmique à la suite d'une mutation ainsi que cela a déjà été évoqué. Mais cette carence peut avoir aussi une raison purement physiologique si l'on admet par exemple que l'évolution oocytaire chez la reine a été perturbée par des conditions environnementales particulières (carence nutritionnelle, perturbation de la colonie, etc.). Dans ce cas, il faut supposer de plus que la déficience induite par cette carence se traduit chez la reine par la pré- 
disposition à pondre des œufs eux-mêmes carencés en vue d'expliquer la transmission de la faiblesse de la colonie d'une génération à la suivante. Telles sont quelques hypothèses parmi d'autres susceptibles d'expliquer la faiblesse persistante de la lignée D. En fait ce phénomène particulier n'est apparu que parce que l'expérimentateur est intervenu. On n'aurait pas pu l'observer dans les conditions habituelles, l'action de la sélection naturelle conduisant à l'extinction rapide de la souche. D'ailleurs il faut remarquer que les techniciens chargés de l'élevage des reines destinées à cette étude s'étaient montrés très réticents quant à l'utilisation du géniteur désigné par le tirage au sort pour la lignée $\mathrm{D}$ et ne s'y étaient résignés que devant les nécessités du plan d'expérience. En effet, cette colonie présentait au début du printemps un faible développement, une évolution lente et un couvain très disséminé, signes très caractéristiques de mauvaise qualité de la reine pour les spécialistes.

Quoi qu'il en soit, nos résultats permettent de comprendre la diversité d'opinions que l'on rencontre dans les milieux apicoles concernant la valeur des hybrides interraciaux puisque nous avons obtenu une forte vigueur hybride dans une lignée et des résultats comparables ou inférieurs aux témoins pour les autres lignées. Ceci est un bon exemple du caractère relativement imprévisible du résultat d'un croisement lorsque ce dernier n'est pas intégré à un plan de sélection adapté. Le présent travail montre donc la nécessité d'une sélection pour la valeur en croisement chez l'abeille.

En effet, si l'on excepte les chercheurs américains (ROBERTS et MACKENSEN 1951, MoEller 1976) qui n'ont pas effectué d'hybrides entre races mais des croisements de lignées consanguines, il n'y a pas eu jusqu'à présent de sélection pour l'aptitude au croisement (FAlCONER 1960) et certaines sont adaptables à l'Abeille mais le plus souvent au prix d'un investissement important.

Revenons à présent à la question initiale : l'évolution des hybrides rétrocroisés sur une race parente. Il résulte de la diversité des lignées qu'une comparaison globale des générations deux à deux est dépourvue de sens. Il semble plus intéressant d'observer le comportement de la lignée $A$ dont la $F_{1}$ très supérieure au témoin manifeste la vigueur hybride rencontrée habituellement dans nos essais. Nous voyons que chaque génération marque une régression d'environ $10 \mathrm{~kg}$ en moyenne sur la précédente ce qui ramène la $F_{2}$ au niveau des témoins et la $F_{3}$ nettement en dessous. La différence entre les témoins et la $F_{3} n$ 'est pas significative mais la puissance du test est affaiblie par le petit nombre de données et la présence d'une donnée quelque peu discordante. Sur ce point donc, ces résultats obtenus chez l'Abeille pour le rendement en miel s'accordent bien à la théorie générale (FALCONER 1960).

Sur le plan pratique, il apparaît nécessaire à l'apiculteur qui utilise des hybrides de veiller au remplacement de ses reines au plus tard à la $\mathrm{F}_{2}$, šil désire ne pas abaisser le niveau de son cheptel. 


\section{ZUSAMMENFASSUNG}

Wegen der Schwierigkeiten, die bei der Selektion der Biene auf Honigertrag auftreten (komplizierte Theorie wegen der besonderen Biologie dieses Tieres, Vorhandensein von Inkompatibilitätsgenen...) hat man schon früh Versuche mit der Hybridisierung verschiedener Rassen unternommen, von denen einige die Bedeutung dieser Technik für die genetische Verbesserung erwiesen haben. Auf Grund des Erfolges, den man mit einer bestimmten Kombination erzielt hat, und zwar (Ligustica $\times$ Caucasica) $\times$ mellifica, sollte die Entwicklung dieses Hybriden im Verlaufe der Rückkreuzungen mit der lokalen Rasse Mellifica verfolgt werden. $\mathrm{Zu}$ diesem $\mathrm{Z}$ weck wurden vier Linien begründet und parallel mit Kontrollvölkern der Rasse $\mathrm{Mel}$ lifica in drei Generationen untersucht (Abb. 1):

$F_{1}$ Königin (Ligustica $\times$ Caucasica) begattet von Mellifica-Drohnen.

$F_{2}$ Tochterkönigin von $F_{1}$, begattet von Mellifica-Drohnen.

$F_{3}$ Tochterkönigin von $F_{2}$, begattet von Mellifica-Drohnen.

Der Vergleich jeder Generation mit der Kontrolle ergibt nur einen einzigen gesicherten Unterschied (die $\mathrm{F}_{7}$-Generation ist der Kontrolle unterlegen). Die Analyse der Tabelle "Generationen $\times$ Linien " gibt einen sehr signifikanten Effekt "Linien ", der den Effekt " Generationen " maskiert; letzterer ist nur in einer einzigen der vier Linien signifikant.

Die starke Variabilität zwischen den Linien ist sehr überraschend, denn die $F_{1}$-Königinnen der verschiedenen Linien sind miteinander verwandt $(\varphi \geqslant 1 / 16)$. Um das Resultat des konstanten Wertes " Null " einer der Linien zu erklären, könnte man an das Auftreten einer schädlichen Mutation in einem Chromosom oder - noch wahrscheinlicher - im Zytoplasma denken oder an eine physiologische Schwäche der Königin, hervorgerufen durch besondere Umweltbedingungen (wie z.B. eine Mangelernährung), die sich auch auf spätere Generationen auswirkt.

Wie auch immer, diese Resultate beweisen die Möglichkeit und die Notwendigkeit einer Selektion auf Kreuzungseignung, sowie den Vorteil eines Austausches der Hybridkönigin spätestens nach der $F_{2^{-}}$ Generation.

\section{BIBLIOGRAPHIE}

Avetissian G. A. et TCherevko 1. A., 1971. - Influence de la consanguinité et de l'hybridation interspécifique sur les caractères des abeilles d'Extrême-Orient. XXIII ${ }^{\circ}$ Congrès International d'Apiculture, Moscou 378-384.

Bilash G. D., Makarov I. I., Sedikh A. V., 1976. - Rejpartition par district des abeilles en U.R.S.S. Symposium de Biologie de l'Abeille. Moscou (U.R.S.S.), Apimondia. Bucarest, Roumanie, 144-154.

FALCONER D. S., 1960. - Introduction to quantitative genetics, Ronald, New York, 365 p.

FRESNAYE J., 1963. - Les erreurs d'orientation des abeilles dans le rucher moderne. Ann. Abeille, 6, 185200.

FresNaye J., 1966. - Cinq années de sélection de l'abeille noire française. Rerue Française d'A piculture, 231, 133-137.

Fresnaye J., 1974. - Biométrie de l'Abeille. Opida, Échauffour, 61370 Ste Gauburge, 42 p.

Fresnaye J., Lavie P., Boesiger E., 1974. - La variabilité de la production du miel chez l'abeille de race noire (Apis mellifica L.) et chez quelques hybrides interraciaux. Apidologie, 5, (1) 1-20.

Fresnaye J., 1975. - Les méthodes d'élevage et la qualité des reines obtenues. Bulletin Technique Apicole OPIDA 2 (2) 15-30.

Fresnaye J., LAVIE P., 1976. - Sélection et hybridation de l'abeille en France. Symp. Génétique, sélection et reproduction de l'abeille. Moscou. Apimonda. Bucarest, 235-24l (et Bul. Tech. Apic. OPIDA 3 (4), (15-20). 
MAUL V., 1972. - Zuchtprogramm mit definierten Sex-Allelen. Comptes rendus du Symposium international de Lunz-am-See (Autriche), Août 1972. Apimonda, Bucarest, Roumanie, 75-79.

Melnitchenko A. N. et Trichina A. S., 1976. - Les bases ecologiques et génetiques de l'hétérosis dans les colonies d'abeilles. Symposium de Biologie de l'abeille. Moscou, Apimondia, Bucarest, Roumanie, 225-231.

Moeller F. E., 1976. - Development of hybrid honey bees. U.S.D.A. 168. Hyattsville, Maryland (U.S.A.).

RAGHIM-ZADE M. S., 1976. - Symposium de Biologie de l'A beille. Moscou (U.R.S.S.), Apimondia, Bucarest, 242-245.

Roberts W. C. et MaCkensen D., 1951. - Breeding improved honeybees. A m. Bee. J. 91 (11), 473-475.

RUTTNER F., 1967. - L'hétérosis chez les abeilles. In LouvEaux J. Les problèmes posès par la génétique et la sélection de l'abeille. Ann. Abeille, 10 (4), 213-252.

Ruttner F., 1968. - L'amélioration du cheptel. In Chauvin R., Trailé de Biologie de l'A beille. Tome IV, 181-236, $1^{\text {re }}$ édit. Masson, Paris.

SNODGRASS R.E., 1956. - Anatomy of the honey bee, Cornell University press. Ithaca. New York, $334 \mathrm{p}$.

VelitchKov V. N., 1976. - Testage des hybrides interraciaux des abeilles. Symposium de Biologie de l'Abeille. Moscou, Apimondia, 232-234.

Woyke J., 1963. - Drone larvae from fertilized eggs of the honeybee. Journat of A picultural Research, 2, $1,19-24$.

Woyke J., 1972. - Sexallèle und kontrollierte Paarung. Comptes rendus du Symposium International de Lunz-am-See (Autriche), Août 1972. Apimondia. Bucarest. Roumanie. 69-74. 